\title{
Current Trends in Functional Imaging of Pheochromocytomas and Paragangliomas
}

\author{
BARRY L. SHULKIN,${ }^{a}$ IOANNIS ILIAS, ${ }^{b}$ JAMES C. SISSON,$^{c}$ \\ AND KAREL PACAK ${ }^{d}$ \\ ${ }^{a}$ Division of Nuclear Medicine, Department of Radiological Sciences, St. Jude \\ Children's Research Hospital, Memphis, Tennessee 38105-2794, USA \\ ${ }^{b}$ Department of Pharmacology, Medical School, University of Patras, \\ Rion-GR-26504, Greece
}

${ }^{c}$ Division of Nuclear Medicine, Department of Radiology, University of Michigan Medical Center, 1500 East Medical Center Drive, UH B1G 505D, Ann Arbor, Michigan 48109-0028, USA

${ }^{d}$ Reproductive Biology and Medicine Branch, National Institute of Child Health and Human Development, National Institutes of Health, Bethesda, Maryland 20892-1109, USA

\begin{abstract}
Most pheochromocytomas/paragangliomas should be evaluated with anatomical imaging (computed tomography or magnetic resonance imaging) followed by functional imaging (nuclear medicine modalities). Functional imaging assures that the tumor is indeed a pheochromocytoma/paraganglioma and enables more thorough localization, especially detecting as many lesions as possible (in particular for metastatic disease). Functional imaging for pheochromocytomas/ paragangliomas, can use radiolabled ligands specific for pathways of synthesis, metabolism, and inactivation of catecholamines or nonspecific ligands. In an overview of the available nuclear medicine modalities, we summarize the accumulated experience and recommend when functional imaging should be applied to patients with pheochromocytoma/paraganglioma.
\end{abstract}

KEYWORDS: nuclear medicine methods; radionuclide imaging; positron emission tomography; pheochromocytoma diagnosis

Address for correspondence: Karel Pacak, M.D., Ph.D., Section on Medical Neuroendocrinology, Reproductive Biology and Medicine Branch, National Institute of Child Health and Human Development, National Institutes of Health, Building 10, CRC, 1 East, Room 1-3140, 10 Center Drive, MSC-1109, Bethesda, MD 20892-1109. Voice: 301-402-4594; fax: 301-402-4712.

e-mail: karel@mail.nih.gov

Ann. N.Y. Acad. Sci. 1073: 374-382 (2006). (C) 2006 New York Academy of Sciences. doi: 10.1196/annals.1353.041 


\section{INTRODUCTION}

Although pheochromocytomas are predominantly sporadic, about $24 \%$ of patients with apparent sporadic pheochromocytoma may be carriers of germline mutations that entail a predisposition for extra-adrenal and often multifocal disease. ${ }^{1}$ About $26-35 \%$ of these tumors are metastastic, ${ }^{2-5}$ and about $10 \%$ present with metastatic disease at initial diagnosis. ${ }^{6}$ In the context of the clinical evaluation of pheochromocytoma/paraganglioma, anatomical imaging with computed tomography (CT) or magnetic resonance (MR) should be done in all patients. After anatomical imaging is performed, functional (nuclear medicine) modalities are used to prove that the tumor is indeed pheochromocytoma/paraganglioma, as well as to detect as many lesions as possible (for metastatic disease) and to choose the appropriate therapeutic plan. Nuclear medicine modalities can be categorized into those that are specific for the catecholamine synthesis/secretion pathway and those that are nonspecific, that is, they reflect other aspects of tumor pathophysiology.

Most pheochromocytoma/paraganglioma cells (chromaffin cells) are distinguished from other tumor cells in that they express the norepinephrine transport system (hNET). ${ }^{7}$ The latter is responsible for the uptake of norepinephrine and epinephrine from the synaptic cleft into the presynaptic neuron of the sympathetic nervous system. hNET thus not only terminates neural transmission by removal of the neurotransmitter norepinephrine but also enables specific functional imaging. In this manuscript we will review the results of imaging of pheochromocytoma obtained with various radiotracers. We begin by describing several radiotracers that depend upon hNET for transport to pheochromocytoma cells followed by tracers whose uptake and retention are independent of hNET (FIG. 1).

\section{SPECIFIC FUNCTIONAL IMAGING}

Attempts to image pheochromocytoma by specific radiotracer techniques began with the development of meta-iodobenzylguanidine (m-IBG) by Wieland and colleagues at the University of Michigan. ${ }^{8}$ Sisson and colleagues reported successful localization of pheochromocytomas using m-IBG. ${ }^{9}$ Initially, $\mathrm{m}-\mathrm{IBG}$ was labeled with iodine-131. $\mathrm{m}$-IBG labeled with iodine-123 has become more common because of the characteristics of I-123, which makes it a better radiolabel than iodine-131 for diagnostic evaluation of pheochromocytoma. Relative to I-131, I-123 has a shorter half-life (13.2 h vs. 8 days), a principal emission photon energy much closer to the $140 \mathrm{kEv}$ of Tc-99m, around which gamma cameras are designed (159 kEv I-123 vs. $364 \mathrm{kEv}$ I-131), and lack of beta particulate emission, which adds to the radiation burden and limits the activity that can be administered. Because of the higher emitted photon flux, I-123 m-IBG images contain about tenfold the number of 


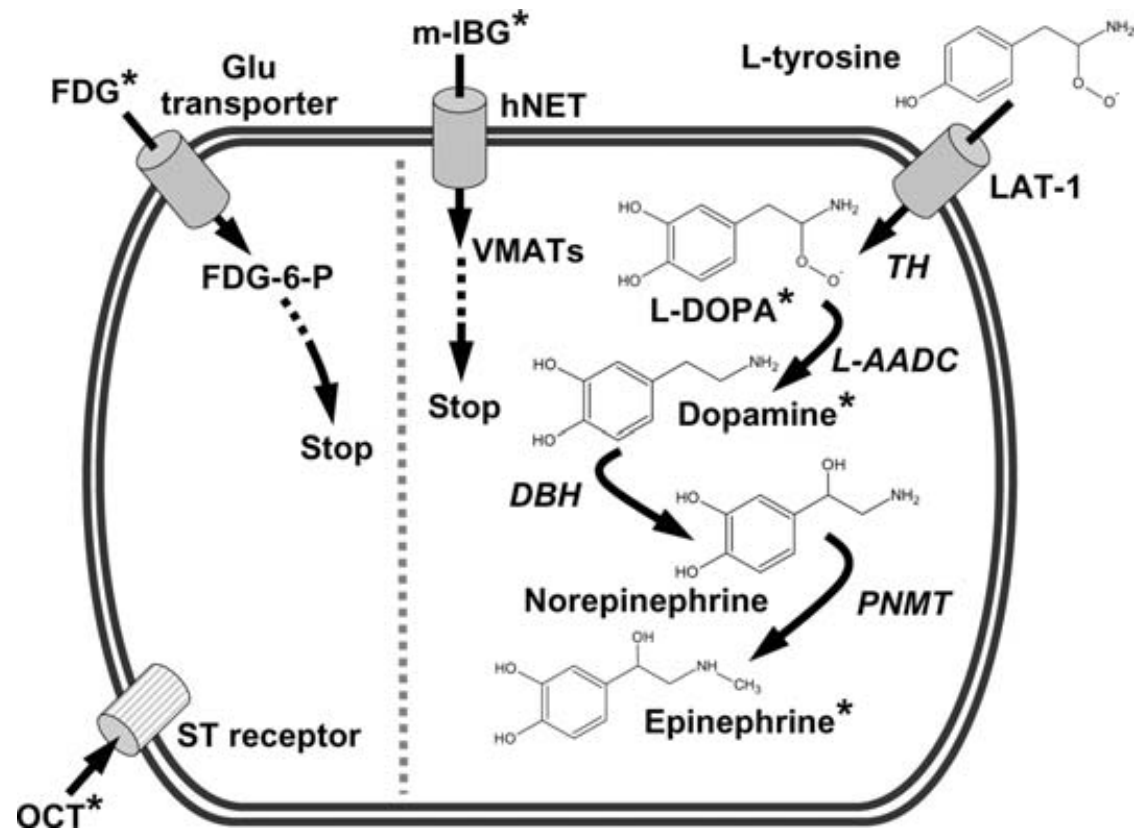

FIGURE 1. Targets for functional imaging of pheochromocytomas/paragangliomas. On the right are shown steps and molecules involved in the synthesis of catecholamines; these are targets for radioligands specific for such cells. On the left are nonspecific targets. Molecules with asterisks are radiolabeled to be used for functional imaging. TH: tyrosine hydroxylase; hNET: human norepinephrine transporter (responsible for re-uptake of catecholamines); m-IBG: meta-iodobenzylguanidine; VMATs: vesicular monoamine transporters (they transport $\mathrm{m}$-IBG into norepinephrine and epinephrine storage vesicles); LAT-1: large neutral amino acid transporter; L-DOPA: L-dihydroxyphenylalanine; LAADC: aromatic-L-amino acid decarboxylase; DBH: dopamine beta-hydroxylase; PNMT: phenylethanolamine-N-methyltransferase; ST: somatostatin; OCT: octreotide; Glu: glucose; FDG: 18-F-fluorodeoxyglucose; FDG-6-P: FDG-6-phosphate; Stop: not further metabolized.

acquired counts as do those obtained with I-131 m-IBG; they are of much higher quality; and tomographic images (SPECT - single photon emission computed tomography) can be obtained for further localization (FIG. 2).

$\mathrm{m}-\mathrm{IBG}$ is useful in localizing pheochromocytomas in any part of the body. It is especially valuable in localizing extra-adrenal sites of pheochromocytoma, and pheochromocytoma in areas of the body previously operated upon, where anatomic imaging is compromised by distortion of anatomy and the presence of metallic clips that degrade anatomic-based images. a-IBG (aminoiodobenzylguanidine) was synthesized as a kit-based formula that would allow local production of the radiolabeled compound. ${ }^{10}$ Although pheochromocytomas were readily identified using this compound, lung accumulation of a-IBG was higher than that of $\mathrm{m}-\mathrm{IBG}$. 

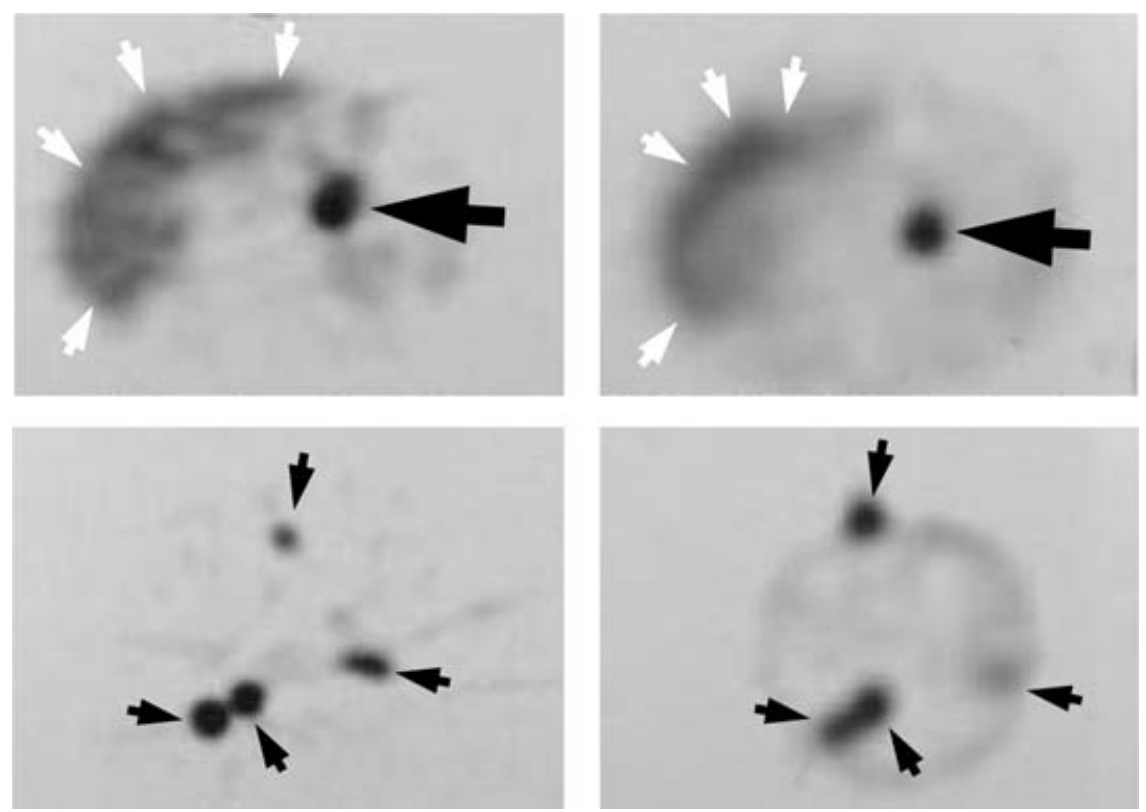

FIGURE 2. The upper left panel is a 11-C-hydroxyephedrine (HED) PET image at 40 $\mathrm{min}$ and the upper right panel is a I-123-m-IBG (meta-iodobenzylguanidine) SPECT image at $24 \mathrm{~h}$ of a 72 -year-old patient with a mid-abdominal pheochromocytoma (hepatic uptake is indicated with small white arrows; the tumor is indicated with the large dark arrow). The lower left panel is a 11-C-HED PET image at $40 \mathrm{~min}$ and the lower right panel is a I-123$\mathrm{m}$-IBG SPECT image at $24 \mathrm{~h}$ of a 60 -year-old patient with malignant pheochromocytoma (multiple foci of uptake are indicated with small dark arrows).

Positron emission tomography (PET) tracers present some advantages compared with single photon-emitting tracers. PET tracers utilize PET cameras, which inherently produce tomographic images with higher spatial resolution than conventional scintigraphic imaging. PET tracers utilize shorter-lived isotopes, enabling higher doses to be administered for a similar radiation exposure. Because of the short half-life, tracers that reflect different aspects of tumor biology could be administered in a single session.

The first positron-emitting tracer of the sympathetic nervous system suitable for administration in humans was m-hydroxyephedrine (HED). HED was designed to image the sympathetic innervation of the heart. Structurally, it resembles norepinephrine but is not susceptible to intracellular degradation by the enzyme monoamine oxidase. m-HED was shown to accumulate in myocardium by transport by the human norepinephrine transporter (hNET). It was labeled with the positron emitter, carbon-11. C-11 has a half-life of only $20 \mathrm{~min}$, thus C-11 HED requires onsite synthesis as its short half-life precludes widespread distribution. Shulkin and colleagues showed that outstanding functional images 
of pheochromocytomas could be obtained using C-11 HED and a PET camera (FIG. 2). ${ }^{11}$ Pheochromocytomas were localized in 9 of 10 patients with pheochromocytoma. High-quality functional images were acquired within minutes of injection, and tumor to nontumor ratios were more favorable than those of I-123 m-IBG. Trampal and colleagues have also reported excellent results with C-11 HED. ${ }^{12}$ Because of the need for onsite synthesis, few centers have access to this compound and it is not yet widely utilized.

Closely related to the endogenous neurotransmitter norepinephrine is the catecholamine epinephrine. Like HED, epinephrine is labeled with C-11, requiring onsite synthesis. Unlike HED, epinephrine is a substrate for the catecholamine catabolic enzymes COMT (catechol- $O$-methyltransferase) and MAO (monoamine oxidase), and thus its retention in myocardium requires intact catecholamine storage vesicles. The majority of pheochromocytomas can be visualized using C-11 epinephrine. ${ }^{13}$ However, the rate of successful localization is less than that of $\mathrm{m}-\mathrm{IBG}$, and it is not likely that $\mathrm{C}-11$ epinephrine will replace $\mathrm{m}$-IBG for functional imaging of pheochromocytoma. As with $\mathrm{C}-11$ HED, uptake occurs quite rapidly and is sustained through the imaging period.

Another PET ligand that targets steps of catecholamine synthesis is F-18fluorodopamine (F-18-DA) (FIG. 3). PET with F-18-DA was performed in 18 patients with metastatic pheochromocytoma and was compared to planar I-131-m-IBG scintigraphy. F-18-DA was superior to I-131-m-IBG: it localized pheochromocytomas in all patients and showed numerous foci that were not imaged with I-131-m-IBG scintigraphy. ${ }^{14}$ In more recent studies, PET with F-18-DA was superior to I-123-m-IBG scintigraphy in imaging adrenal and/or benign pheochromocytomas and paragangliomas (Pacak et al., unpublished data). ${ }^{15}$

Fluorodopa was developed to image the dopaminergic elements of the central nervous system. More recently it has been shown to provide functional images of pheochromocytoma. In 14 patients, 17 sites of pheochromocytoma were identified both by MRI and PET F-18 fluorodopa imaging. ${ }^{16}$ In 4 patients, $\mathrm{m}$-IBG scintigraphy was falsely negative. F-18 fluorodopa is presumably converted into fluorodopamine and then transported into pheochromocytomas by hNET, although the large neutral amino acid transporter may also contribute.

\section{NONSPECIFIC FUNCTIONAL IMAGING}

FDG (18-fluorodeoxyglucose) is used for the detection and management of many types of tumors in children and adults. FDG portrays the glucose metabolic activity of tumors, and its uptake and retention are unrelated to catecholamine uptake and storage. FDG is now widely available commercially. The first report of FDG uptake in pheochromocytoma involved two patients whose pheochromocytomas did not accumulate mIBG. Further studies showed that most benign and most malignant pheochromocytomas could be localized 

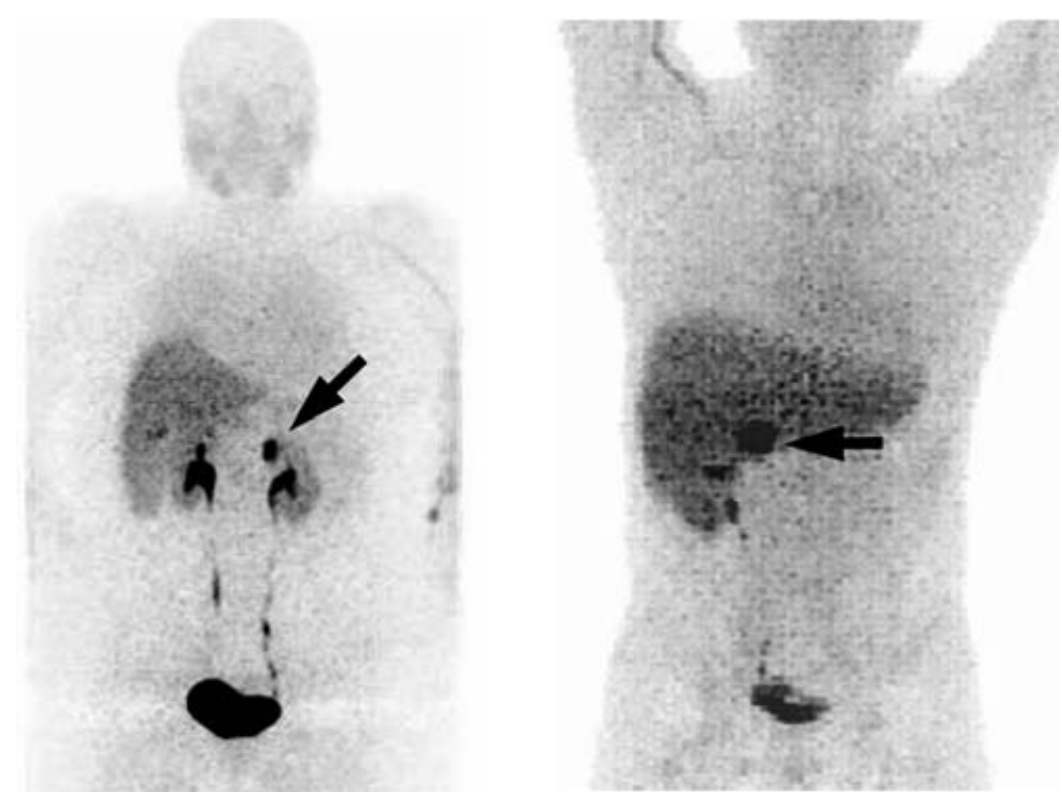

FIGURE 3. PET scans with F-18-fluorodopamine of patients with adrenal pheochromocytomas: left panel shows a left adrenal pheochromocytoma (dark arrow) and right panel shows a right adrenal pheochromocytoma (dark arrow).

with FDG PET. ${ }^{17}$ FDG PET appears particularly valuable for the localization of pheochromocytomas that do not accumulate m-IBG or other tracers of the sympathetic nervous system. ${ }^{17-19}$

Somatostatin receptor scintigraphy is of limited value in the localization of pheochromocytoma. Both pheochromocytomas and paragangliomas express somatostatin receptors. ${ }^{20-22}$ The octapeptide octreotide is an analogue of somatostatin that is chelated with diethylenetriaminepentaacetate (DTPA) and labeled with indium-111 for diagnostic imaging. We have not found it useful in the evaluation of pheochromocytomas in the abdomen because of its normal intense accumulation in the liver, spleen, and kidneys, and excretion into the gastrointestinal tract. It might be useful for identifying malignant/metastatic pheochromocytomas or paragangliomas; ${ }^{23-29}$ such malignant/metastatic tumors can be candidates for radiolabeled somatostatin analogue therapy.

\section{NEWER FUNCTIONAL IMAGING LIGANDS}

Newer potential functional imaging modalities specific for pheochromocytomas/paragangliomas include PET with F-18-fluorobenzylguanidine.${ }^{30} \mathrm{New}$ somatostatin receptor ligands, which are yet to be evaluated clinically, for 
SPECT $^{31}$ or $\mathrm{PET}^{32-37}$ have been developed (they are nevertheless nonspecific for pheochromocytomas/paragangliomas).

\section{RECOMMENDATIONS AND PERSPECTIVES}

In conclusion, regarding clinical practice, functional imaging evaluation should be done after anatomical imaging with CT or MRI. Furthermore, we propose that functional imaging be performed in all patients with extra-adrenal, metastatic, or multiple pheochromocytomas, norepinephrinesecreting pheochromocytomas, and epinephrine-secreting adrenal pheochromocytomas larger than $5 \mathrm{~cm}$ in diameter. Additionally, functional imaging modalities should be chosen in the postsurgical setting, when biochemical evaluation for pheochromocytoma is inconclusive and in particular when anatomical imaging is negative. Specific functional imaging modalities (m-IBG scintigraphy or PET with 18-F-DA, 18-F-DOPA, or 11-C-HED) are to be used first, and if these turn out to be negative, nonspecific modalities (somatostatin receptor scintigraphy or FDG-PET) should follow.

In future, we anticipate that at centers with PET departments and access to specific PET ligands, most functional imaging procedures for pheochromocytoma/paraganglioma may be PET studies.

\section{REFERENCES}

1. Neumann, H.P. et al. 2002. Germ-line mutations in nonsyndromic pheochromocytoma. N. Engl. J. Med. 346: 1459-1466.

2. Mundschenk, J. et al. 1998. Malignant pheochromocytoma. Exp. Clin. Endocrinol. Diabetes 106: 373-376.

3. AvERBUCH, S.D. et al. 1988. Malignant pheochromocytoma: effective treatment with a combination of cyclophosphamide, vincristine and dacarbazine. Ann. Intern. Med. 109: 267-273.

4. Proye, C.et al. 1992. High incidence of malignant pheochromocytoma in a surgical unit: 26 cases out of 100 patients operated from 1971 to 1991. J. Endocrinol. Invest. 15: 651-663.

5. Tischler, A. 1998. The adrenal medulla and extra-adrenal paraganglia. In Functional Endocrine Pathology. K. Kovacs \& S. Asa, Eds.: 550-595. Blackwell. Oxford.

6. GoldSTEIN, R.E. et al. 1999. Clinical experience over 48 years with pheochromocytoma. Ann. Surg. 229: 755-766.

7. Schulz, C. et al. 2004. Principles of catecholamine biosynthesis, metabolism and release. Front. Horm. Res. 31: 1-25.

8. WiELAND, D.M. et al. 1980. Radiolabeled adrenergic neuron-blocking agents: adrenomedullary imaging with $\left[{ }^{131} \mathrm{I}\right]$ iodobenzylguanidine. J. Nucl. Med. 21: 349-353.

9. Sisson, J.C. et al. 1981. Scintigraphic localization of pheochromocytoma. N. Engl. J. Med. 305: 12-17. 
10. ShUlKIN, B. et al. 1986. 123I-4-Amino-3-iodobenzylguanidine (123I-AIBG), a new sympatho-adrenal imaging agent: comparison with 123I-metaiodobenzylguanidine (123I-MIBG). J. Nucl. Med. 27: 1138-1142.

11. SHULKIN, B.L. et al. 1992. PET scanning with hydroxyephedrine: an approach to the localization of pheochromocytoma. J. Nucl. Med. 33: 1125-1131.

12. Trampal, C. et al. 2004. Pheochromocytomas: detection with ${ }^{11} \mathrm{C}$ hydroxyephedrine PET. Radiology 230: 423-428.

13. SHULKIN, B.L. et al. 1995. PET epinephrine studies of pheochromocytoma. J. Nucl. Med. 36: 22P-23P.

14. ILIAS, I. et al. 2003. Superiority of 6-[ $\left[{ }^{18} \mathrm{~F}\right]$-fluorodopamine positron emission tomography versus $\left[{ }^{131} \mathrm{I}\right]$-metaiodobenzylguanidine scintigraphy in the localization of metastatic pheochromocytoma. J. Clin. Endocrinol. Metab. 88: 40834087.

15. ILIAS, I. et al. 2004. Comparison of $6-\left[{ }^{18} \mathrm{~F}\right]$-fluorodopamine positron emission tomography with [123In]-metaiodobenzylguanidine and [111In]-pentetreotide scintigraphy in the localization of pheochromocytoma, ENDO. 86th Annual Meeting of the American Endocrine Society, New Orleans, LA, The Endocrine Society: $452-453$.

16. Hoegerle, S. et al. 2002. Pheochromocytomas: detection with ${ }^{18} \mathrm{~F}$ DOPA whole body PET-initial results. Radiology 222: 507-512.

17. ShUlKIn, B.L. et al. 1999. Pheochromocytomas: imaging with 2-[fluorine18]fluoro-2-deoxy-D-glucose PET. Radiology 212: 35-41.

18. Mamede, M. et al. 2005. Discordant localization of [18-F]-fluorodeoxyglucose, in [18-F]-fluorodopamine-negative metastatic pheochromocytoma sites. J. Nucl. Med. 46 (CD-ROM Suppl.): 1324.

19. EzUdDIN, S. et al. 2005. MIBG and FDG PET findings in a patient with malignant pheochromocytoma: a significant discrepancy. Clin. Nucl. Med. 30: 579581.

20. Hofland, L.J. et al. 1999. Immunohistochemical detection of somatostatin receptor subtypes sst1 and sst2A in human somatostatin receptor positive tumors. J. Clin. Endocrinol. Metab. 84: 775-780.

21. ReuBI, J.C. et al. 1992. In vitro and in vivo detection of somatostatin receptors in pheochromocytomas and paragangliomas. J. Clin. Endocrinol. Metab. 74: 10821089.

22. MundSCHENK, J. et al. 2003. Somatostatin receptor subtypes in human pheochromocytoma: subcellular expression pattern and functional relevance for octreotide scintigraphy. J. Clin. Endocrinol. Metab. 88: 5150-5157.

23. VAN DER HARST, E. et al. 2001. [(123)I]metaiodobenzylguanidine and [(111)In] octreotide uptake in benign and malignant pheochromocytomas. J. Clin. Endocrinol. Metab. 86: 685-693.

24. SCHMIDT, M. et al. 2002. Clinical value of somatostatin receptor imaging in patients with suspected head and neck paragangliomas. Eur. J. Nucl. Med. Mol. Imaging 29: $1571-1580$.

25. LAMBERTS, S.W. et al. 1990. Somatostatin-receptor imaging in the localization of endocrine tumors. N. Engl. J. Med. 323: 1246-1249.

26. KRENNING, E.P. et al. 1993. Somatostatin receptor scintigraphy with [111In-DTPAD-Phe1]- and [123I-Tyr3]-octreotide: the Rotterdam experience with more than 1000 patients. Eur. J. Nucl. Med. 20: 716-731.

27. TelisCHI, F.F. et al. 2000. Octreotide scintigraphy for the detection of paragangliomas. Otolaryngol. Head Neck Surg. 122: 358-362. 
28. Duet, M. et al. 2003. Clinical impact of somatostatin receptor scintigraphy in the management of paragangliomas of the head and neck. J. Nucl. Med. 44: 1767-1774.

29. Bustillo, A. et al. 2004. Octreotide scintigraphy in the head and neck. Laryngoscope 114: 434-440.

30. BERRY, C.R. et al. 2002. Imaging of pheochromocytoma in 2 dogs using p-[18F] fluorobenzylguanidine. Vet. Radiol. Ultrasound. 43: 183-186.

31. PlachcinskA, A. et al. 2003. Clinical usefulness of 99mTc-EDDA/HYNIC-TOC scintigraphy in oncological diagnostics: a preliminary communication. Eur. J. Nucl. Med. Mol. Imaging 30: 1402-1406.

32. HofmanN, M. et al. 2001. Biokinetics and imaging with the somatostatin receptor PET radioligand (68)Ga-DOTATOC: preliminary data. Eur. J. Nucl. Med. 28: $1751-1757$.

33. Anderson, C.J. et al. 2001. 64Cu-TETA-octreotide as a PET imaging agent for patients with neuroendocrine tumors. J. Nucl. Med. 42: 213-221.

34. WESTER, H.J. et al. 2003. PET imaging of somatostatin receptors: design, synthesis and preclinical evaluation of a novel $18 \mathrm{~F}$-labelled, carbohydrated analogue of octreotide. Eur. J. Nucl. Med. Mol. Imaging 30: 117-122.

35. Schottelius, M. et al. 2004. First (18)F-labeled tracer suitable for routine clinical imaging of sst receptor-expressing tumors using positron emission tomography. Clin. Cancer Res. 10: 3593-3606.

36. Kowalski, J. et al. 2003. Evaluation of positron emission tomography imaging using [68Ga]-DOTA-D Phe(1)-Tyr(3)-octreotide in comparison to [111In]DTPAOC SPECT: first results in patients with neuroendocrine tumors. Mol. Imaging Biol. 5: 42-48.

37. GABRIEL, M. et al. 2003. An intrapatient comparison of 99mTc-EDDA/HYNICTOC with 111In-DTPA-octreotide for diagnosis of somatostatin receptorexpressing tumors. J. Nucl. Med. 44: 708-716. 